\title{
Auxetic structures from 3D printed hybrid textiles
}

\author{
Subin Shajoo ${ }^{1}$, David Schmelzeisen ${ }^{2}$, Christopher Pastore ${ }^{3, *}$ \\ ${ }^{1}$ Department of Design, Politecnico di Milano, Italy. \\ 2 Institut für Textiltechnik der RWTH Aachen University (ITA), Germany \\ ${ }^{3}$ Thomas Jefferson University, Philadelphia, USA \\ * Corresponding author E-mail address: Chris.Pastore@Jefferson.edu
}

\section{INFO}

CDAPT, ISSN 2701-939X

Peer reviewed article

2021, Vol. 2, No. 1, pp. 91-102

DOI 10.25367/cdatp.2021.2.p91-102

Received: 16 July 2021

Accepted: 10 August 2021

Available online: 14 August 2021

\section{Keywords}

Auxetic materials,

3D printed textiles,

4D textiles

\begin{abstract}
Auxetic structures have been produced using $3 D$ printing and knitted textile materials. A review of other auxetic textiles is presented along with the new materials. A range of configurations were developed, prototyped, and tested to demonstrate significant auxetic response, including Poisson's ratio up to negative one. The concept of $4 D$ textiles was employed to create environmentally responsive hinges in some structures, allowing the material to change shape in response to thermal stimulus.
\end{abstract}

(C) 2021 The authors. Published by CDAPT. This is an open access article under the CC BY-NC-ND license https://creativecommons.org/licenses/ peer-review under responsibility of the scientific committee of the CDAPT.

(C) 2021 CDAPT. All rights reserved.

\section{Introduction}

When a material is subject to an applied tensile extension in a given direction $\left(\varepsilon_{\mathrm{a}}\right)$, the material will demonstrate an extension in the other orthogonal, transverse, directions $\left(\varepsilon_{t}\right)$. Poisson's ratio $v$ for a given material is defined as the negative ratio of these two values [1]: $v=-\varepsilon_{\mathrm{t}} / \varepsilon_{\mathrm{a}}$. For most materials, Poisson's ratio is positive. There are a few materials that have negative Poisson's ratios, as a result of their molecular structure. These tend to be highly porous foam-like structures [2]. In fact, the behavior comes from the microstructural geometry of the foam [3]. Materials with negative Poisson's ratios are called auxetic.

Expanding the auxetic microstructure to a larger scale, there is a well-established field of research to evaluate structural systems that demonstrate auxetic behavior through the use of structural elements at a much larger scale than molecules or pores, by combining triangles [4], squares [5], or auxetic hexagons [6].

There are a range of applications for auxetic materials, including biomedical [7], sports [8], and textiles [9]. One of the interesting properties is the ability of auxetic materials and structures to form synclastic shapes (including domes and spheres), as opposed to traditional materials which form anticlastic 
(positive Poisson's ratio) or even monoclastic surfaces (high shear modulus), as illustrated in Fig. 1. A synclastic curve is formed from an increase in dimensions in both direction and shearing of the surface, monoclastic requires no stretch and no shear, and anticlastic one has shearing as well as an increased length in one direction and decreased length in the other.

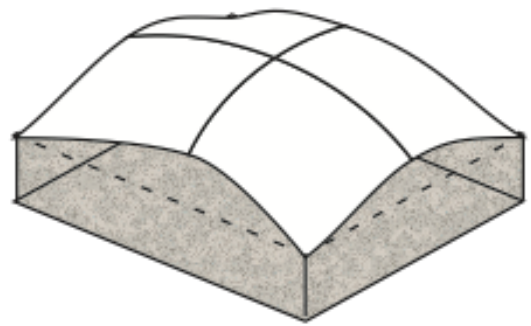

Synclastic

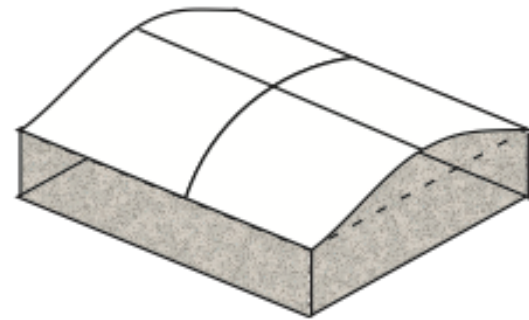

Monoclastic

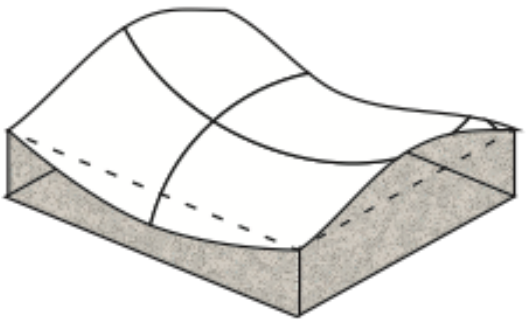

Anticlastic

Fig. 1. Classification of curved surfaces as synclastic, monoclastic, or anticlastic.

Design and modeling of novel auxetic materials and structures is a developing field. In this paper, we are examining a methodology for creating auxetic membrane structures using 3D printing combined with textile materials. The result is a quick method of producing auxetic structures that are durable and suitable for a range of applications.

\section{Auxetic shapes}

The classic example of an auxetic structure is a hinged hexagonal system. If we consider a framework produced in a hexagonal, honey-comb type of shape, assuming it is a pin jointed truss where the members are stiff and the joints have no resistance to rotation, we create a structure that has a traditional behavior, as shown in the top of Fig. 2 [10].
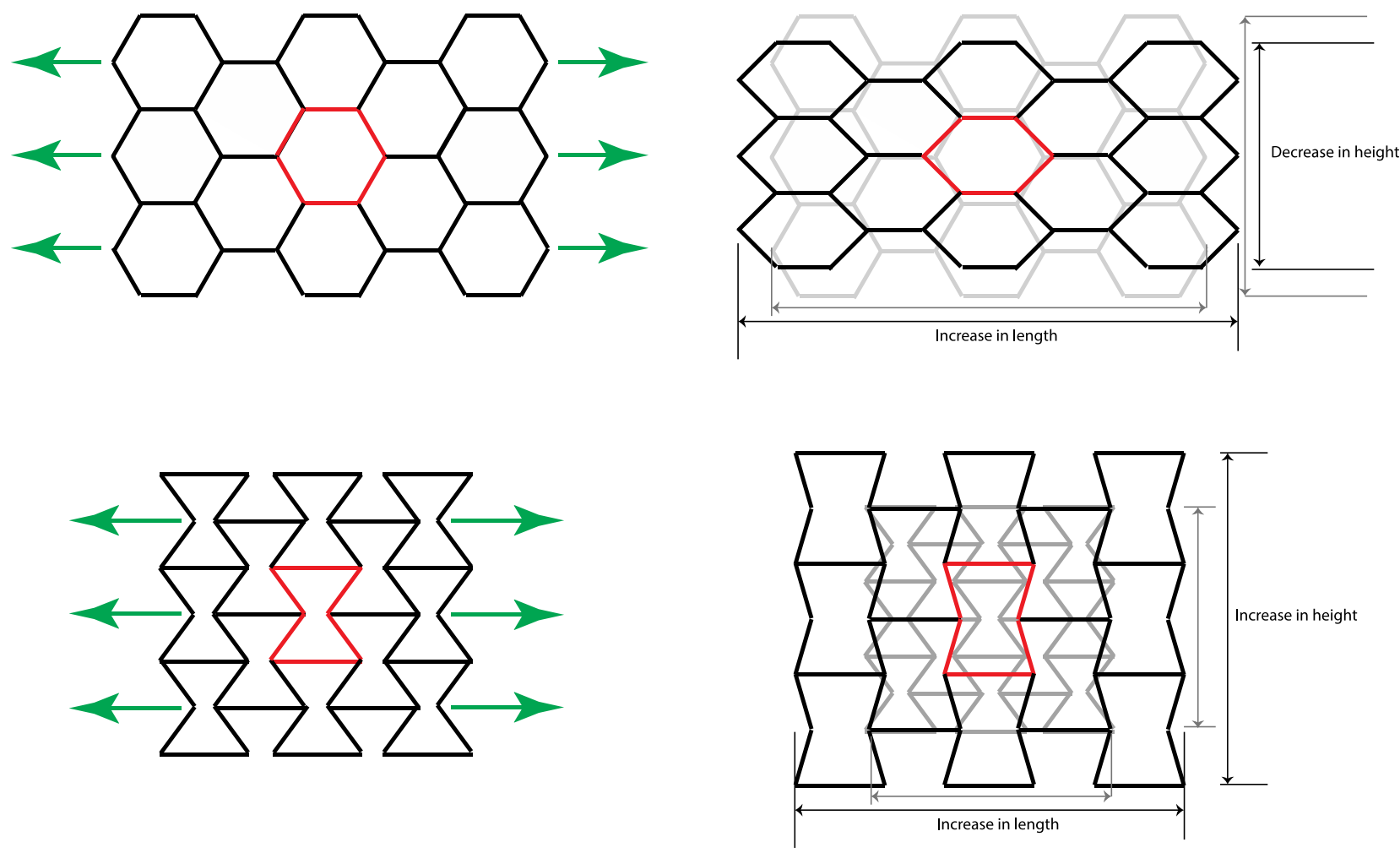

Fig. 2. Comparison of traditional honeycomb shape with positive Poisson's ratio (top) and an auxetic structure formed from an inward facing hexagon demonstrating a negative Poisson's ratio, thus an auxetic material (bottom). 
But changing that hexagon to a convex, inward facing one, as illustrated in the bottom of Fig. 2, the behavior of the structure changes dramatically. This is due to the orientation of the structural elements. In order for the inward facing arms of the hexagons to extend horizontally, they must rotate around their joints, resulting in an increase in the height of the structure. The specific Poisson's ratio of this structure depends on the relative length of the horizontal elements to diagonal elements, and the angle of orientation of the inward facing diagonals.

It should be noted that this behavior is easy to demonstrate and model for an idealized (perfect) truss with no resistance to joint rotation [10]. In an actual material system, the behavior is not perfect.

There are many different geometries that can accomplish auxetic structural behavior. The key component is the rotation of individual structural elements that interact with each other to create the auxetic behavior.

Investigators have considered a range of possible systems, such as star shaped inclusions, to create auxetic composites [11], interlocking hexagons [6], or trilobal inclusions in foam [8], or other types of "missing rib" structures embedded in foam [12].

\subsection{Auxetic yarns}

Auxetic behavior has also been demonstrated in linear systems, such as yarns [13]. The principle here is a bit different than the auxetic structures identified above. The idea is to make a yarn structure that increases in average diameter when stretched in the axial direction. This is accomplished through the use of two different yarn systems, one notably stiffer than the other. In a structure often termed helical auxetic yarn [14], or HAY, the stiff element is wrapped around a core of the less stiff element, as shown in Fig. 3. Upon application of axial load, the stiffer wrapper resists elongation and uses the applied energy to move to the center rather than extend. The lower modulus core element can deform and move into an outer spiral.
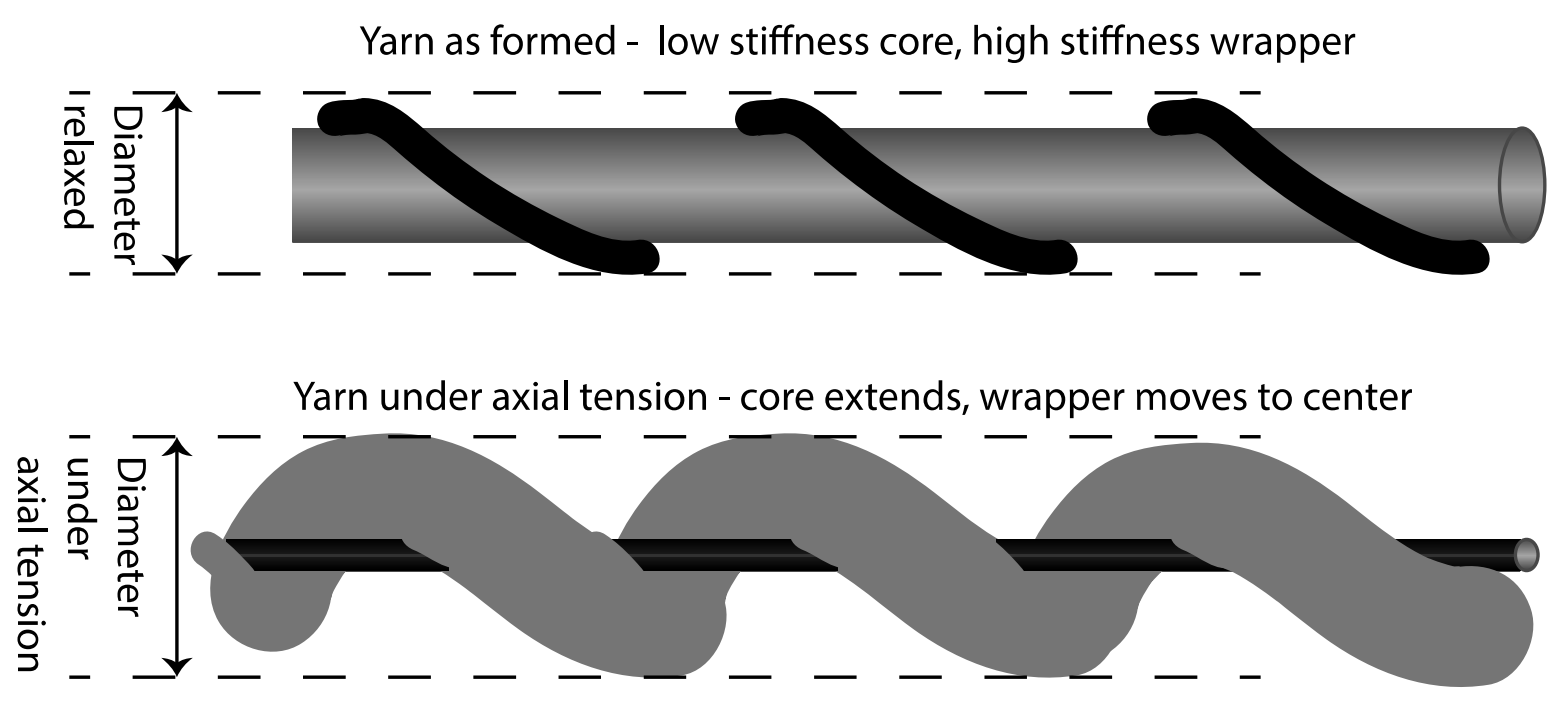

Fig. 3. Schematic illustration of an auxetic yarn (helical auxetic yarn) showing relaxed state (top) with a low stiffness core and a high stiffness wrapper, the same yarn under axial tension (bottom) wherein the low stiffness core has stretched and moved to the outside while the high modulus wrapper moves to the center, creating an increase in the effective diameter of the yarns.

The exchange of positions between core and wrapper result in an auxetic yarn, i.e. in an increase in the effective diameter of the yarn. In general terms, the effective diameter of the relaxed yarn $D_{r}$ can be estimated in terms of the core diameter, $d_{c}$, and the wrapper diameter, $d_{w}$ :

$$
D_{r}=d_{c}+2 d_{w}
$$


When it is fully extended, the diameter $\mathrm{D}_{\mathrm{e}}$ can be approximated as

$$
D_{e}=2 d_{c}+d_{w}
$$

Thus, the diameter of the composite yarn varies by approximately $\left(\mathrm{d}_{\mathrm{c}}-\mathrm{d}_{\mathrm{w}}\right)$, depending on the applied load. The magnitude of that change depends on the starting diameters of the two elements.

It is important to note that, as illustrated in the drawings, this is the effective diameter. The entire volume is not filled with yarn, but rather the projection of the diameter along the axis of the yarn shows the auxetic behavior.

\subsection{Auxetic textiles}

There is also a significant amount of research in ways to create auxetic textiles [15,16]. One approach is creating fabrics using the HAY systems described above. This is not a particularly effective method, as the yarns themselves, carrying the load, will try to resist the negative Poisson effect, as illustrated in Fig. 4. Further, the yarns in the perpendicular direction will resist the expansion in that direction.

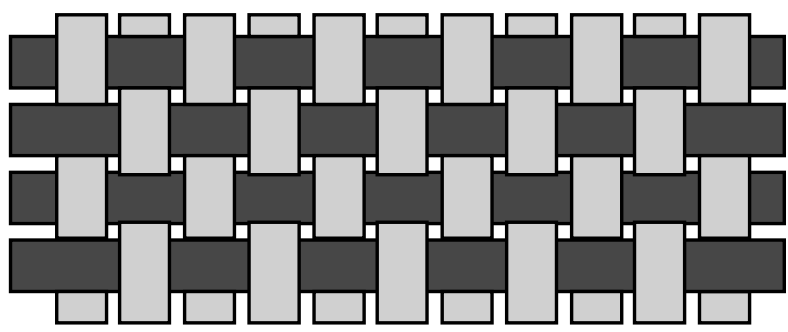

Relaxed fabric with HAY yarns

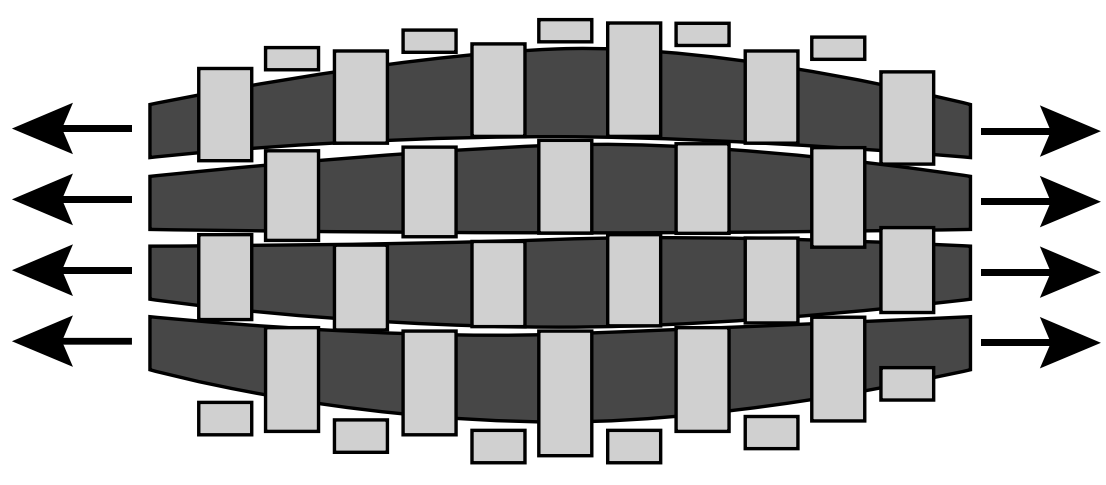

Loaded fabric with HAY yarns

Fig. 4. Schematic illustration of a woven fabric with HAY yarns, relaxed in the top image and under load in the bottom image.

When the yarns in Fig. 4 expand due to the negative Poisson's ratio, they need to move out of their original position because of the expansion of the neighboring yarns. In order to carry the load, the individual yarns must be held in some position. Thus, the motion of the expanding yarns attempts to make a curved path rather than the original straight path. This creates compressive forces at the sides of the yarn and these forces attempt to reduce the expansion of the yarns.

Although woven fabrics made from HAY yarns are not particularly auxetic in practice, they do change permeability and porosity significantly when under load making them good for applications such as blast protection [14]. 
A number of researchers have explored the potentials of incorporating auxetic shapes (see above) into warp knitted fabrics so that the yarns formed into auxetic patterns $[17,18]$. These structures appear to provide the auxetic behavior, relying on the use of elastic yarns in addition to stiffer yarns to make the transition. Most of the configurations only have the negative Poisson effect in the first loading, and are not reversible [19]. However, some work has been done attempting to create reversible warp knitted fabrics that demonstrate auxetic behavior [20].

Nonwoven fabrics can be designed to produce auxetic behavior in the thickness direction only through proper needle punching [21].

A different approach to inducing auxetic behavior in textile structures is through folding techniques, inspired by origami arts [22-24]. The basic concept is to make the fabric in a highly folded configuration and in effect the material oriented in thickness direction reorients into the transverse direction, resulting in a negative Poisson's ratio in the transverse planar direction, but a highly positive value in the thickness direction [25]. A typical origami folding pattern is shown in Fig. 5. This technique has been used to fold textiles, holding the creases through an ironing or pressing procedure. Variations on the folding patterns exist, and all generally can create auxetic behavior in the plane of the folded system.

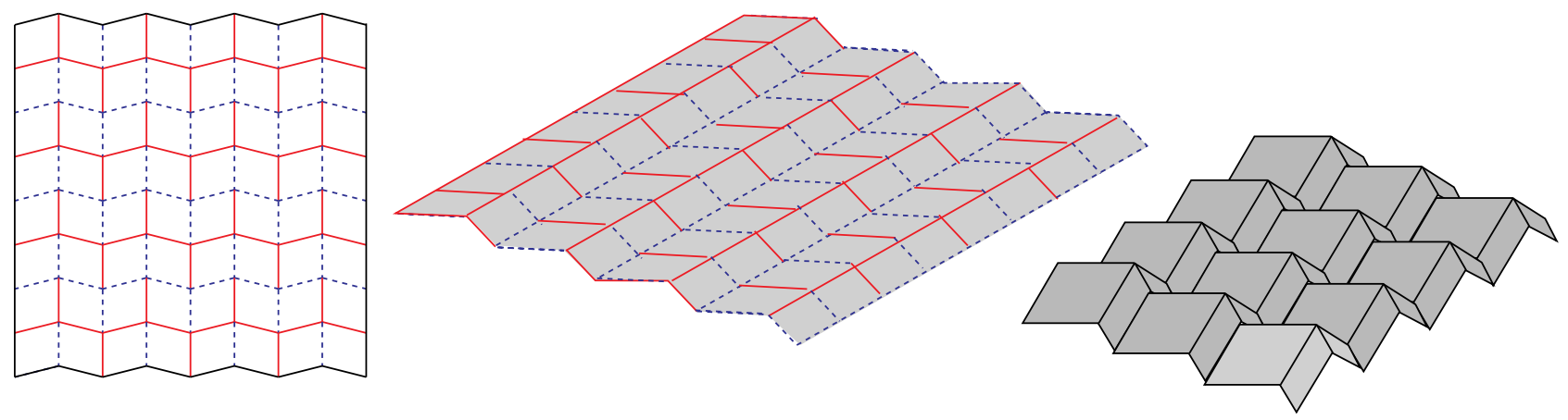

Fig. 5. Schematic illustration of an origami folding pattern that creates auxetic structures used for paper or textile materials.

\section{3D printing on textiles}

There has been a rapid growth in research regarding 3D printing in recent years driven by the low cost and material efficiency of the technology. Combining 3D printing and textile materials is still a relatively new research area [26-28]. This method offers the promise of "4D Textiles" - hybrid textile/3D printed structures that can change structural form with time $[29,30]$. The adhesion between substrate and printed polymer is important for long-term stability of the developed structure [31].

Because the textile material is highly flexible, as opposed to the relative stiffness of the printed polymer, in the hybrid system the textile acts as a unifying hinge and structural reinforcement that can permit and restrain both flexure and torsion.

In the process described here, an elastic knitted fabric was used as the textile substrate. This allows prestretching of the fabric prior to printing, in which case the textile is a repository of stored elastic energy. This method of storing energy is commonly employed in 4D textiles [32], typically to create curvature or to provide a mechanism for assisting shape transformation. In the process described, a pre-strain level of $50 \%$ in two orthogonal directions was applied to the knitted fabric prior to printing. This opens the pores of the fabric, but also provides some energy that pulls the structure towards the center, as illustrated in Fig. 6. 


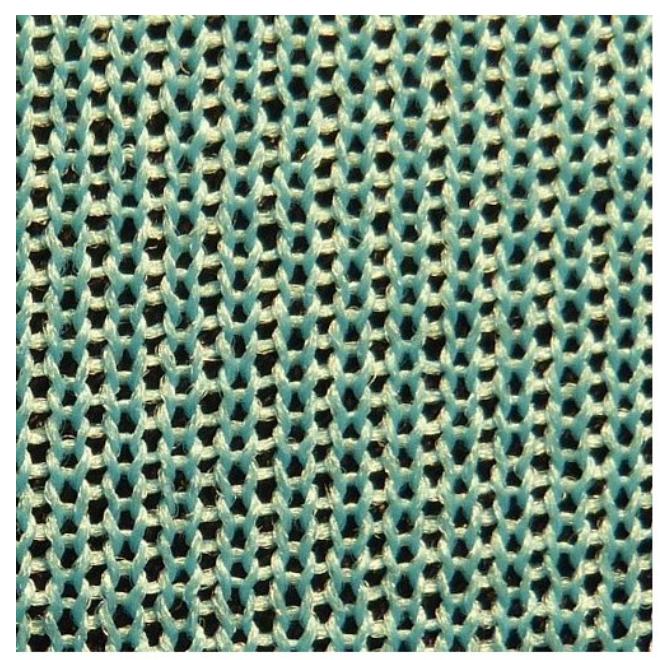

Jersey knit, no stretch

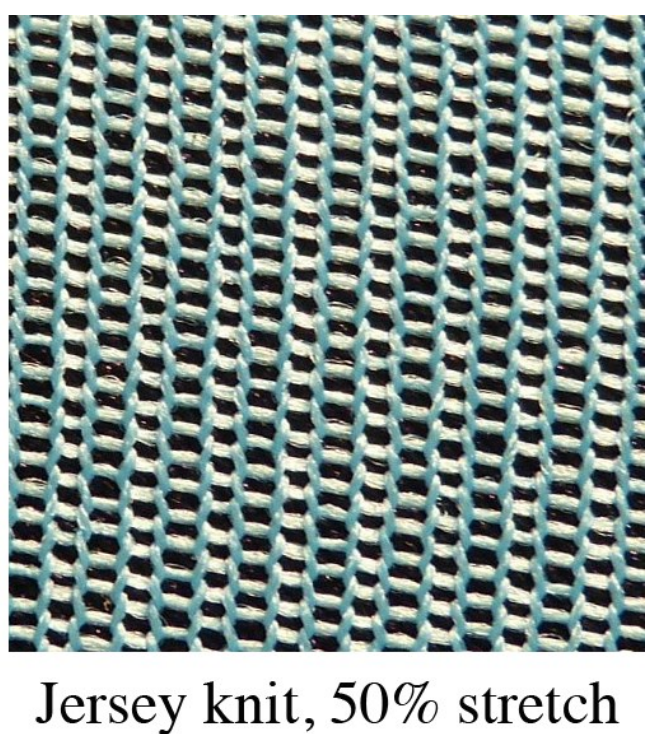

Fig. 6. Photomicrograph of elastic Jersey knit with no stretch (left) and after $50 \%$ stretch in both $x$-and $y$-directions (right).

The fabric stretch is maintained during printing with a flat metallic plate that has pins at the sides, as shown in Fig. 7. The fabric is measured and marked prior to stretching on the plate to ensure the proper dimensions are achieved. The pins do not provide a perfect boundary condition, as can be seen, but near the center of the fabric the strain field is relatively uniform as was demonstrated in Fig. 6.

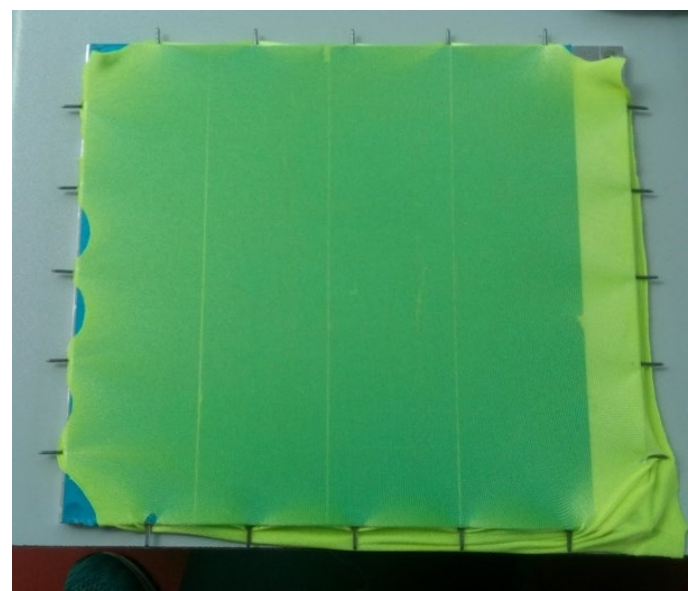

Fig. 7. Weft knitted fabric stretched and held in place on a plate prior to 3D printing. Pins at the edges of the plate hold the fabric still during the processing.

In this research, the polymer was printed onto the fabric surface using a Mass Portal Pharaoh $\mathrm{XD} \otimes$ printer using a $0.4 \mathrm{~mm}$ nozzle. This printer uses $1.75 \mathrm{~mm}$ thermoplastic filaments with two filament feeds and a heater capable of printing at $300{ }^{\circ} \mathrm{C}$. The auxetic forms used for printing were designed with SolidWorks ${ }^{\circledR}$. After exporting them as STL files, G-codes of the sliced model was developed using Simplify $3 \mathrm{D}^{\circledR}$. During the slicing process, all printing parameters, such as printing velocity, layer thickness, flow rate, etc. could be defined. The G-codes were sent to the 3D printer and used to print the structure. Details of these printing parameters are contained in Appendix A.

\subsection{Auxetic structures on textile substrates}

For example, as illustrated in Fig. 8, a structure such as the corner joined squares could be printed onto a textile material. The flexible nature of the textile should allow the structural shapes to create the auxetic behavior. The initial goal was to develop a 3D print/textile hybrid that demonstrated the auxetic behavior shown below. 


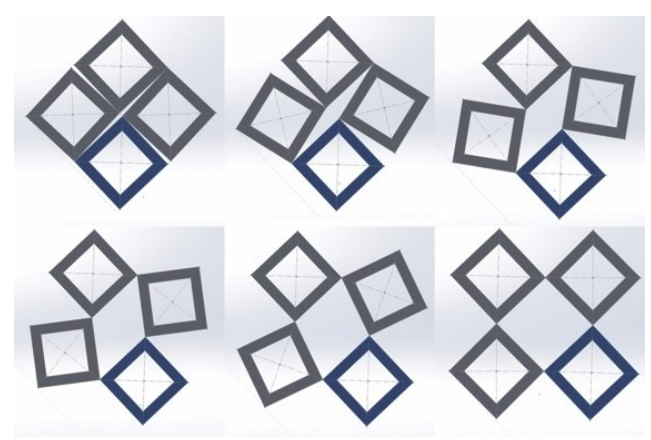

Fig. 8. Simulation of rectangular auxetic structures joined at corners showing auxetic in-plane behavior.

The 3D print/textile hybrid requires two elements - the 3D printing design and execution and the selection of an appropriate textile substrate. In order to get this structure printed onto a textile, it is necessary to create a model of the print. There are several critical aspects of the print model:

- Design a joint for the corner which is capable of repeated flexing.

- Ensure the polymer bonds well with the textile substrate.

- Provide sufficient stiffness to the frame element.

To accomplish this, the design employed used two different polymers during the printing process. A polylactic acid (PLA) was used as a soft material to provide the corner hinge. This was also used as a base layer for the entire structure because the low viscosity PLA provides a good infiltration with the textile substrate.

For the structural square, acrylonitrile butadiene styrene (ABS) was printed. This has a much higher stiffness and higher glass transition temperature than the PLA. The print pattern was two layered - first a layer of PLA and then a selective layer of ABS, as illustrated in Fig. 9. During printing, the ABS and PLA were each provided in the form of a filament with diameter of $1.75 \mathrm{~mm}$.

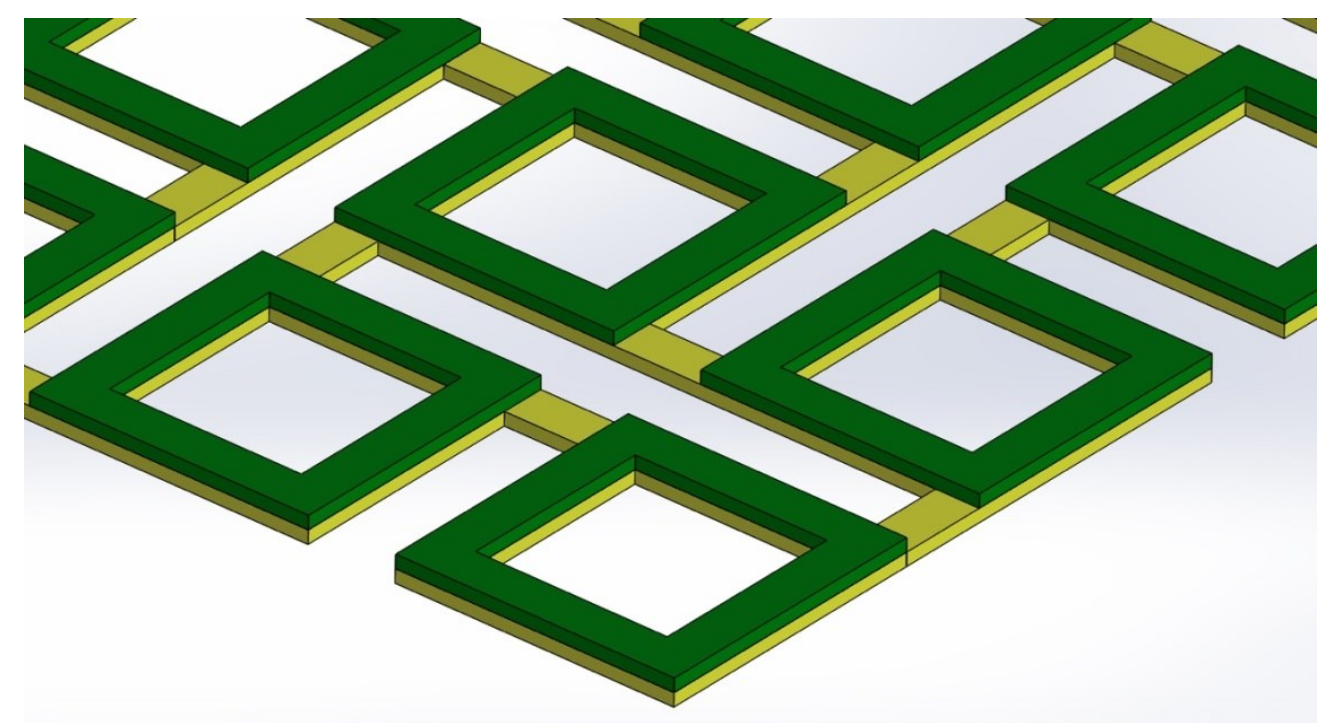

Fig. 9. CAD drawing used to control the printer for the two-layer system. In this illustration dark green represents the $A B S$ and light green represents the PLA.

As mentioned previously, the substrate needs to be able to rotate at the joint. The localized shear strain field on the textile will be quite high, and thus a highly extensible, low shear modulus fabric is needed. An elastic jersey knit, formed from polyester yarns with Spandex, was used. This fabric has low shear modulus, high extensibility, and good elastic recovery.

Initial experiments involved printing acrylonitrile butadiene styrene (ABS) onto the pre-stretched jersey knit fabric (formed of polyester/Spandex yarns). After printing rectangular shapes onto the fabric, the 
fabric was slit selectively to allow the fabric to function as a rotational hinge for the structure. If the fabric was not slit, the tensile modulus of the fabric would fight against the expansion in the transverse direction. By slitting the fabric, the knit functions primarily as the hinge material in addition to providing a protective membrane inside the printed squares.

A sample of a $3 \times 3$ printed auxetic square structure is illustrated in Fig. 10. The resulting structure had a very strong auxetic behavior, and a tendency to returning to the original form. However, repeated loadings resulted in delamination of the ABS from the knit fabric, particularly at the corners of the squares where stresses are highest.

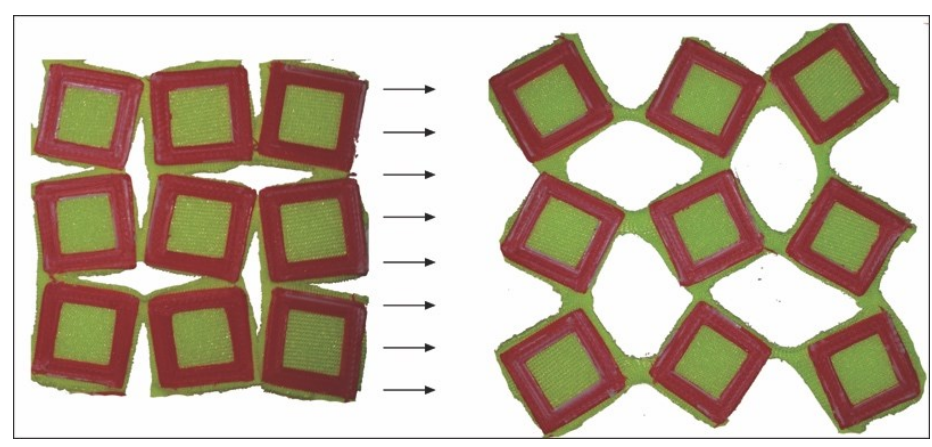

Fig. 10. Experimental study of a $3 \times 3$ rectangular auxetic formed from ABS printed onto polyester/Spandex jersey knit fabric. The fabric was slit to create space for rotation (left) and after extension the auxetic behavior is quite clear and dramatic (right).

Larger structures using smaller squares were also explored. Fig. 11 shows a $7 x 7$ print, again slit between the squares, demonstrating significant auxetic behavior and also the ability to form into synclastic surfaces. The Poisson's ratio calculated from the example below is -0.9.

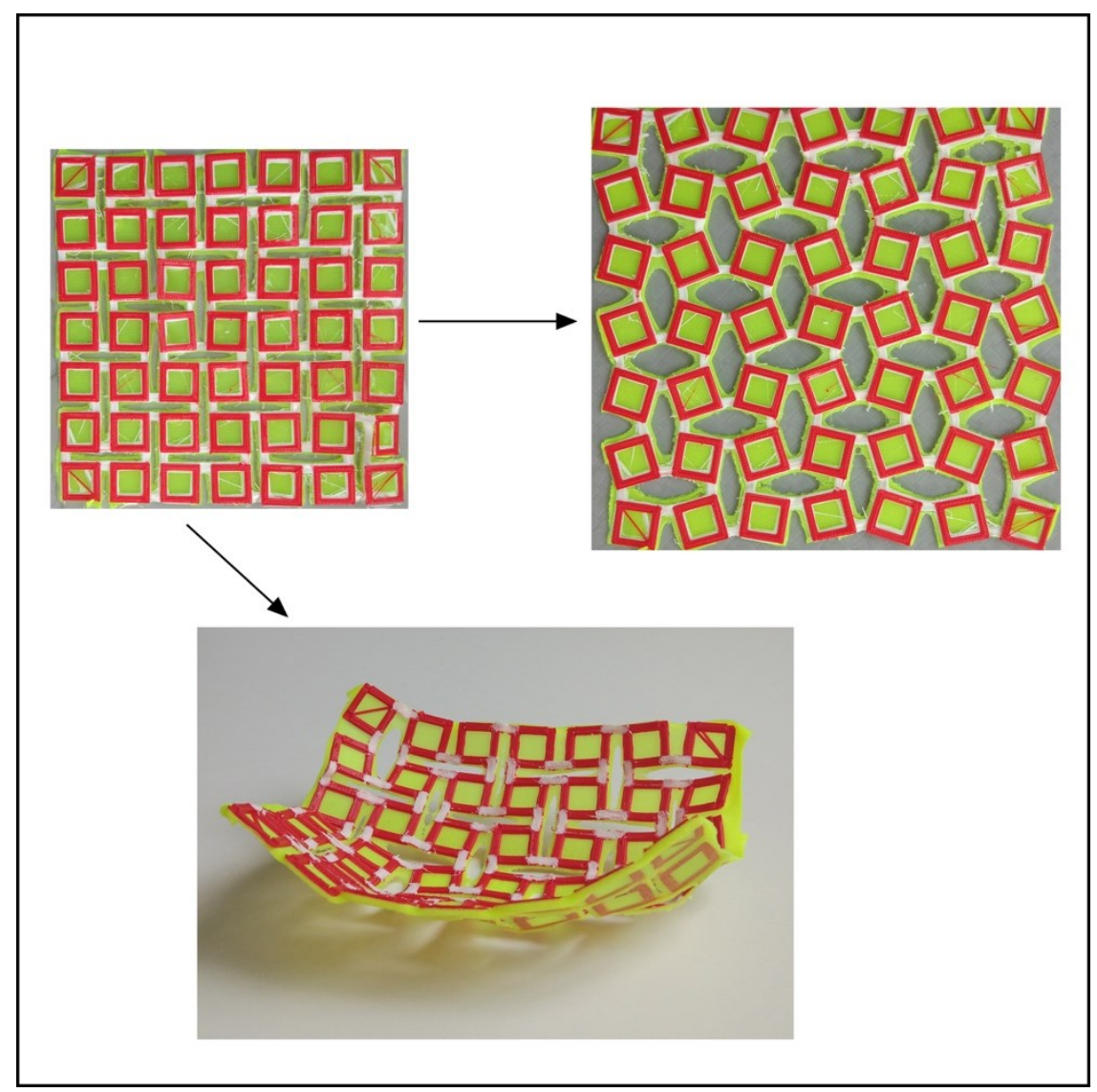

Fig. 11. $7 x 7$ printed auxetic square structure on elastic jersey knit showing auxetic behavior with a Poisson's ratio of -0.9 (right) and also ability to form synclastic surfaces (bottom). 
An alternative triangular auxetic form was also printed. The fabric was slit at the sides of the triangles to allow maximum extension in the transverse direction. The auxetic textile shown in Fig. 12 demonstrated a Poisson's ratio of -1.05 under simple extension.
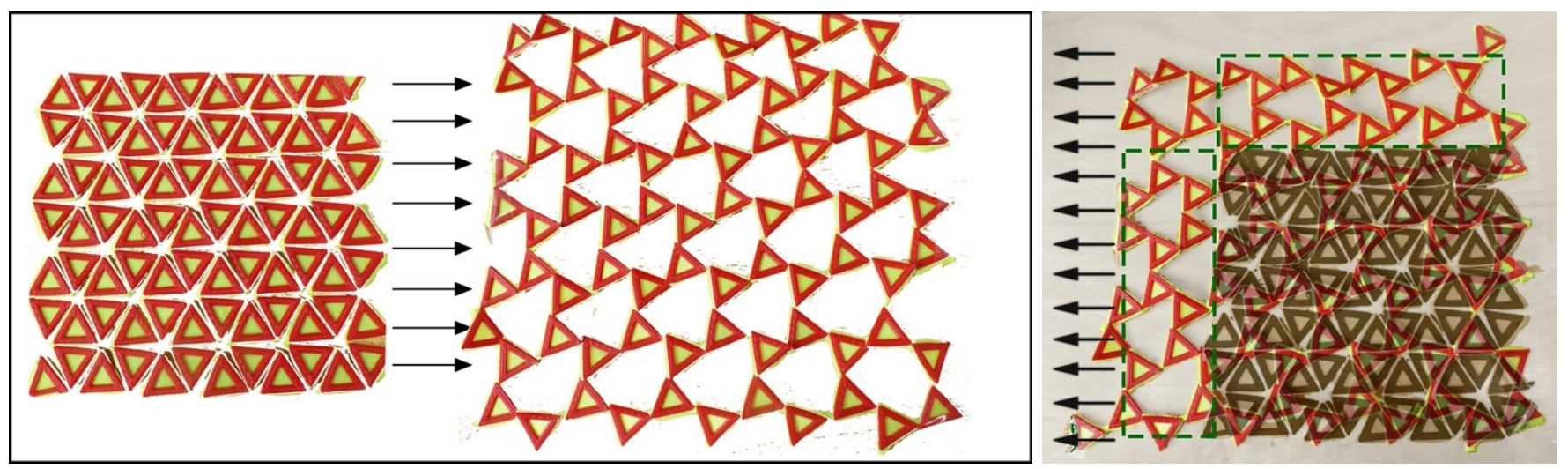

Fig. 12. Triangular auxetic structure printed onto elastic jersey knit showing an auxetic behavior with a Poisson's ratio of -1.05 .

\subsection{Triggered response}

The use of PLA as the hinge material provides an additional interesting feature potential. Because the PLA has a relatively low glass transition temperature $T_{G}$, approximately $60-65^{\circ} \mathrm{C}$, it is possible to heat the PLA above $T_{G}$ but still stay below the $T_{G}$ of the ABS (which is approximately $105^{\circ} \mathrm{C}$ ). When above $T_{G}$, the PLA has extremely low modulus and can be deformed to a very high level. The structure can be heated above the PLA $T_{G}$, deformed, and then cooled. In this way the structure will maintain the deformed shape to a large extent.

In this way, one can manufacture a wrap which can form synclastic shapes when heated above $60{ }^{\circ} \mathrm{C}$ and retain that shape when cooled. This could be useful for any number of wraps, including casts for limbs, molds, prosthesis and more. Particularly when a synclastic shape is desired, these auxetic textile hybrids can provide such geometries without wrinkling or folding.

The wrap formed in this way will also return back to its original shape on heating, allowing reusability. When the structure is heated above the PLA $T_{G}$, the PLA acts as a rubbery material, enabling the squares to freely rotate. When the structure heated without applying any external force, the stored energy in the fabric will return it back to the original printed shape. Fig. 13 shows a time sequence of an auxetic textile hybrid. The original structure is that shown in Fig. 11 - a $7 \times 7$ printed square system. The hybrid was heated above $60^{\circ} \mathrm{C}$ and extended then cooled. The image shown in the top left of Fig. 13 is the structure at room temperature. The PLA "froze" into place in the extended form. Warm water $\left(60^{\circ} \mathrm{C}\right)$ was poured over the hybrid system and the next 7 images show the system over a period of less than one minute. The PLA gets above its $T_{G}$ and then the stored energy in the knitted substrate returns the structure to its original shape. A reusable, synclastic structure is thus produced. 

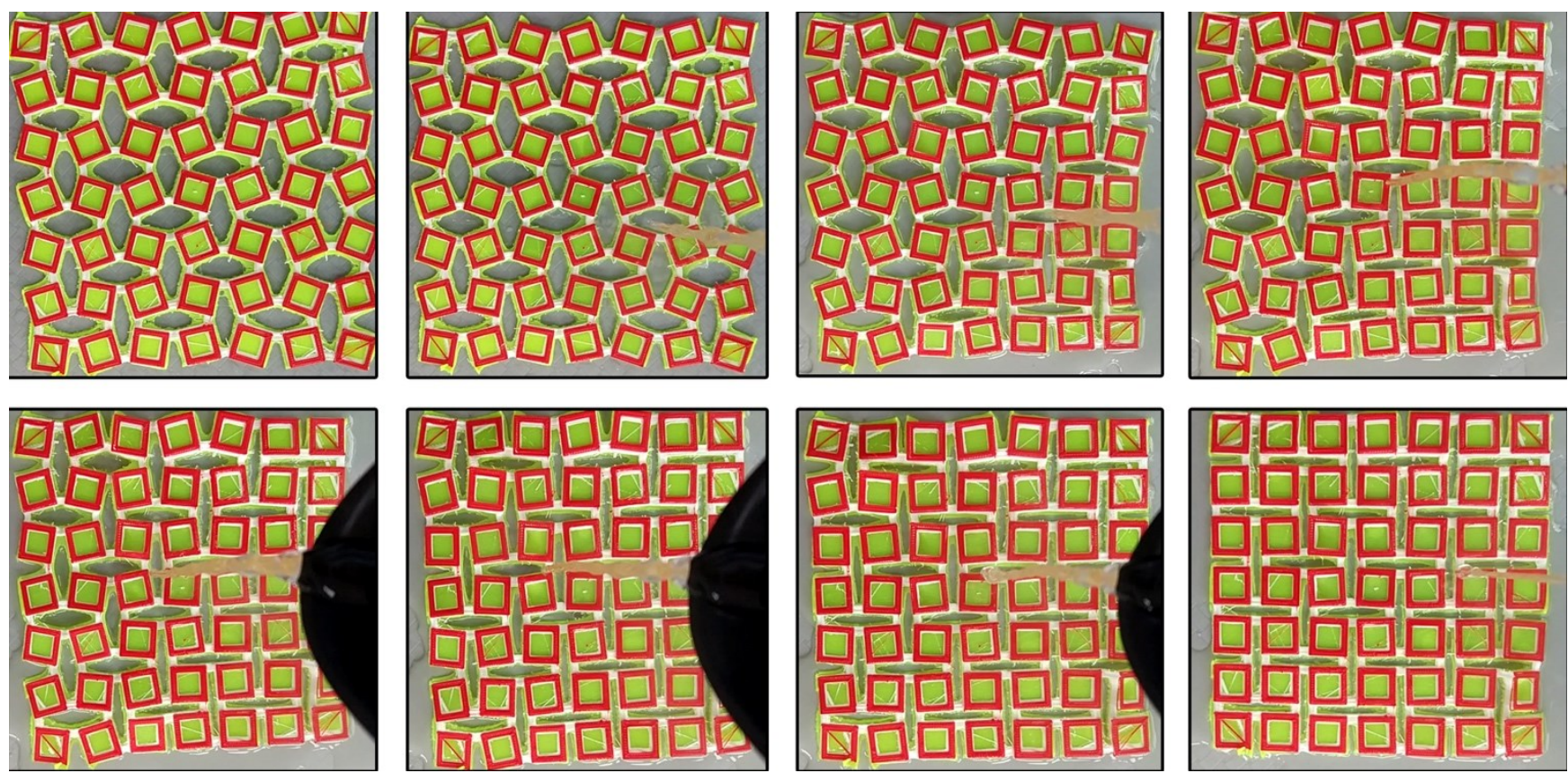

Fig. 13. A time sequence of an auxetic textile hybrid that was frozen into an extended shape (top left) and then introduced to $60^{\circ} \mathrm{C}$ water. As the images progress from left to right on top, and then on the bottom from left to right, the structure changing shape back to the as-printed form can be seen.

\section{Conclusion}

Initially two different auxetic forms, rotating squares and rotating triangles, were printed on the prestrained fabric to study the auxetic properties. Slits were made on the fabric in such a way that the fabric would act as a hinge for the rotating shapes. Rotating squares were chosen based on the ease to design and make slits.

PLA hinges were added to the structure because for a structure to function as a support wrap, it was necessary that wrap retained the necessary shape. The low glass transition temperature of PLA enabled the structure to change shape or form synclastic shapes when heated to temperature of $65^{\circ} \mathrm{C}$ because at temperatures above $60^{\circ} \mathrm{C}$ the modulus of rigidity of the PLA falls, enabling the squares to rotate freely when stretched.

\section{References}

[1] S.D. Poisson. 1827. Note sur l'Extension des Fils et des Plaques élastiques. Annales de Chimnie et Physique 36, 384-387.

[2] R. Lakes. 1987. Foam Structures with a Negative Poisson's Ratio. Science 235, 4792, 1038.1040. DOI: https://doi.org/10.1126/science.235.4792.1038.

[3] K. E. Evans. 1991. Auxetic polymers: a new range of materials. Endeavour 15, 4, 170-174. DOI: https://doi.org/10.1016/0160-9327(91)90123-S.

[4] J.N. Grima and K.E. Evans. 2006. Auxetic behavior from rotating triangles. Journal of Materials Science 41, 10, 3193-3196. DOI: https://doi.org/10.1007/s10853-006-6339-8.

[5] J.N. Grima and K.E. Evans. 2000. Auxetic behavior from rotating squares. Journal of Materials Science Letters 19, 17, 1563-1565. DOI: https://doi.org/10.1023/A:1006781224002.

[6] N. Ravirala, A. Alderson, and K.L. Alderson. 2007. Interlocking hexagons model for auxetic behaviour. Journal of Materials Science 42, 17, 7433-7445. DOI: https://doi.org/10.1007/s10853-007-1583-0.

[7] M. Mir, M. N. Ali, J. Sami, and U. Ansari. 2014. Review of Mechanics and Applications of Auxetic Structures. Advances in Materials Science and Engineering 2014, 753496. DOI: https://doi.org/10.1155/2014/753496.

[8] M. Sanami, N. Ravirala, K. Alderson, and A. Alderson. 2014. Auxetic Materials for Sports Applications. Procedia Engineering 72, 453-458. DOI: https://doi.org/10.1016/j.proeng.2014.06.079.

[9] Z. Wang and H. Hu. 2014. Auxetic materials and their potential applications in textiles. Textile Research Journal 84, 15, 1600-1611. DOI: https://doi.org/10.1177/0040517512449051. 
[10] Y. Prawoto. 2012. Seeing auxetic materials from the mechanics point of view: A structural review on the negative Poisson's ratio. Computational Materials Science 58, 140-153. DOI: https://doi.org/10.1016/j.commatsci.2012.02.012.

[11] G. E. Stavroulakis. 2005. Auxetic behaviour: appearance and engineering applications. physica status solidi (b) 242, 3, 710-720. DOI: https://doi.org/10.1002/pssb.200460388.

[12] C. W. Smith, J. N. Grima, and K. E. Evans. 2000. A novel mechanism for generating auxetic behaviour in reticulated foams: missing rib foam model. Acta Materialia 48, 17, 4349-4356. DOI: https://doi.org/10.1016/S1359-6454(00)00269-X.

[13] M. R. Sloan, J. R. Wright, and K. E. Evans. 2011. The helical auxetic yarn - A novel structure for composites and textiles; geometry, manufacture and mechanical properties. Mechanics of Materials 43, 9, 476-486. DOI: https://doi.org/10.1016/j.mechmat.2011.05.003.

[14] J. R. Wright, M. K. Burns, E. James, M. R. Sloan, and K. E. Evans. 2012. On the design and characterisation of low-stiffness auxetic yarns and fabrics. Textile Research Journal 82, 7, 645-654. DOI: https://doi.org/10.1177/0040517512436824.

[15] D. Rant, T. Rijavec, and A. Pavko-Çuden. 2013. Auxetic Textiles. Act Chimica Slovenica 60, 4, 715-723.

[16] S. C. Ugbolue, Y. K. Kim, S. B. Warner, Q. Fan, C.-L. Yang, O. Kyzymchuk, and Y. Feng. 2010. The formation and performance of auxetic textiles. Part I: theoretical and technical considerations. Journal of the Textile Institute 101, 7, 660-667. DOI: https://doi.org/10.1080/00405000902733790.

[17] C. Ugbolue, S., K. Kim, Y. and B. Warner, S. 2012. Engineered Warp Knit Auxetic Fabrics. Journal of Textile Science \& Engineering 2, 1, 1000e103. DOI: https://doi.org/10.4172/2165-8064.1000e103.

[18] M. Starbuck, S. C. Anand, N. Ravirala, K. Alderson, and A. Alderson, 2008. Fabrics having knit structures exhibiting auxetic properties and garments formed thereby. Patent No. US 20080011021 A1, Jan. 17, 2008.

[19] Z. Wang and H. Hu. 2017. Tensile and forming properties of auxetic warp-knitted spacer fabrics. Textile Research Journal 87, 16, 1925-1937. DOI: https://doi.org/10.1177/0040517516660889.

[20] S. C. Anand and D. Skertchly. 2010. Auxetic knitted fabric. Patent No. WO 2010125397 A1, Nov. 4, 2010.

[21] P. Verma, M. L. Shofner, A. Lin, K. B. Wagner, and A. C. Griffin. 2015. Inducing out-of-plane auxetic behavior in needle-punched nonwovens. physica status solidi (b) 252, 7, 1455-1464. DOI: https://doi.org/10.1002/pssb.201552036.

[22] S.M. Felton, M. T. Tolley, B. Shin, C. D. Onal, E. D. Demaine, D. Rus, and R. J. Wood. 2013. Self-folding with shape memory composites. Soft Matter 9, 32, 7688. DOI: https://doi.org/10.1039/c3sm51003d.

[23] E. A. Peraza-Hernandez, D. J. Hartl, R. J. Malak Jr,, and D. C. Lagoudas. 2014. Origami-inspired active structures: A synthesis and review. Smart Materials and Structures 23, 9, 094001. DOI: https://doi.org/10.1088/0964-1726/23/9/094001.

[24] V. Turk. 2015. Space Origami Could Pack Big Structures into Tiny Satellites. Retrieved August 14, 2021 from https://www.vice.com/en/article/ezp4mm/space-origami-could-pack-big-structures-into-tiny-satellites.

[25] Y. Liu, H. Hu, J. K. C. Lam, and S. Liu. 2010. Negative Poisson's Ratio Weft-knitted Fabrics. Textile Research Journal 80, 9, 856-863. DOI: https://doi.org/10.1177/0040517509349788.

[26] G. J. Brinks, M. M. C. Warmöskerken, R. Akkerman, and W. Zweers. 2013. The added value of 3D polymer deposition on textiles. Proc. of $13^{\text {th }}$ AUTEX World Textile Conference, Dresden, Germany.

[27] E. Pei, J. Shen, and J. Watling. 2015. Direct 3D printing of polymers onto textiles: Experimental studies and applications. Rapid Prototyping Journal 21, 5, 556-571. DOI: https://doi.org/10.1108/RPJ-09-2014-0126.

[28] L. Sabantina, F. Kinzel, A. Ehrmann, and K. Finsterbusch. 2015. Combining 3D printed forms with textile structures - mechanical and geometrical properties of multi-material systems. IOP Conference Series: Materials Science and Engineering 87, 012005. DOI: https://doi.org/10.1088/1757-899X/87/1/012005.

[29] D. Schmelzeisen, H. Koch, and C. Pastore. 2018. 4D-Textiles: Hybrid textile structures that change structural form with time. In Y. Kyosev, Mahltig, B., and A. Schwarz-Pfeiffer (Eds.). Narrow Fabrics and Smart Textiles. Springer International Publishing.

[30] K. Simonis, D. Schmelzeisen, V. Gesché, and T. Gries. 2017. 4D textiles: application in sports industry. Future Textiles 2, 2, 38-39.

[31] A. Narula, C. M. Pastore, D. Schmelzeisen, S. El Basri, J. Schenk, and S. Shajoo. 2018. Effect of knit and print parameters on peel strength of hybrid 3-D printed textiles. Journal of Textiles and Fibrous Materials 1 251522111774925. DOI: https://doi.org/10.1177/2515221117749251.

[32] R. Melnikova, A. Ehrmann, and K. Finsterbusch. 2014. 3D printing of textile-based structures by fused deposition modelling (FDM) with different polymer materials. IOP Conference Series: Materials Science and Engineering 62, 012018. DOI: https://doi.org/10.1088/1757-899X/62/1/012018. 


\section{Appendix A. Printing Parameters}

The printing parameters used for this project are contained in the table below. There were slight differences in the settings used for the ABS and PLA materials.

\begin{tabular}{|c|c|c|c|}
\hline printMaterial & PLA & ABS & units \\
\hline extruderName & Extruder 1 & Extruder 2 & \\
\hline extruderToolheadNumber & 0 & 1 & \\
\hline extruderDiameter & 0.4 & 0.4 & $\mathrm{~mm}$ \\
\hline extruderWidth & 0.4 & 0.4 & $\mathrm{~mm}$ \\
\hline extrusionMultiplier & 0.9 & 1 & \\
\hline extruderUseRetract & 1 & 1 & \\
\hline extruderRetractionDistance & 1 & 1 & \\
\hline extruderExtraRestartDistance & 0 & 0 & \\
\hline extruderRetractionZLift & 0 & 0 & \\
\hline extruderRetractionSpeed & 1800 & 1800 & \\
\hline extruderUseCoasting & 0 & 0 & \\
\hline extruderCoastingDistance & 0.2 & 0.2 & $\mathrm{~mm}$ \\
\hline layerHeight & 0.2 & 0.2 & $\mathrm{~mm}$ \\
\hline topSolidLayers & 3 & 3 & \\
\hline bottomSolidLayers & 3 & 3 & \\
\hline perimeterOutlines & 2 & 2 & \\
\hline firstLayerHeightPercentage & 90 & 100 & \\
\hline firstLayerWidthPercentage & 100 & 100 & \\
\hline firstLayerUnderspeed & 0.5 & 0.5 & \\
\hline internallnfillPattern & Rectilinear & Rectilinear & \\
\hline externalInfillPattern & Rectilinear & Rectilinear & \\
\hline infillPercentage & 20 & 20 & \\
\hline outlineOverlapPercentage & 15 & 15 & \\
\hline infillExtrusionWidthPercentage & 100 & 100 & \\
\hline minlnfillLength & 5 & 5 & \\
\hline infillLayerInterval & 1 & 1 & \\
\hline infillAngles & 45 & 45 & \\
\hline SetpointTemperatures & 190 & 225 & ${ }^{\circ} \mathrm{C}$ \\
\hline fanLayers & 2 & 1 & \\
\hline fanSpeeds & 100 & 0 & \\
\hline defaultSpeed & 3600 & 5400 & \\
\hline outlineUnderspeed & 0.5 & 0.5 & \\
\hline solidInfillUnderspeed & 0.8 & 0.8 & \\
\hline supportUnderspeed & 0.8 & 0.8 & \\
\hline rapidXYspeed & 4800 & 4800 & \\
\hline rapidZspeed & 1000 & 1000 & \\
\hline
\end{tabular}

